\title{
Equation of state of strongly interacting matter in compact stars
}

\author{
A. Lavagno ${ }^{1,2}$ and G. Pagliara ${ }^{1,3, a}$ \\ 1 Dipartimento di Fisica, Politecnico di Torino, 10129 Torino, Italy \\ 2 INFN, Sezione di Torino, 10125 Torino, Italy \\ 3 INFN, Sezione di Ferrara, 44100 Ferrara, Italy
}

Received: 17 June 2005 /

Published online: 21 March 2006 - (c) Società Italiana di Fisica / Springer-Verlag 2006

\begin{abstract}
We study the equation of state of strongly interacting matter at large densities and vanishing temperature. The hadronic matter equation of state is computed in a relativistic mean-field model and the quark matter equation of state is computed using a NJL-type model which takes into account the possibility of formation of the gapless color flavor locked phase. We focus in particular on the possible phase transition from hadronic matter to quark matter using both Maxwell and Gibbs constructions. We finally discuss the relevance of the equation of state in the context of compact stars and we propose some astrophysical signatures of the presence of quark matter in compact stars.
\end{abstract}

PACS. 26.60.+c Nuclear matter aspects of neutron stars - 25.75.Nq Quark deconfinement, quark-gluon plasma production, and phase transitions

\section{Introduction}

The equation of state of strongly interacting matter at densities below the saturation density of nuclear matter $\rho_{0}=0.16 \mathrm{fm}^{-3}$ is relatively well known $[1,2]$ due to the large amount of experimental data on nuclear physics available. At larger densities there are many uncertainties due to the lack of experimental data; the saturation of nuclear force makes, in fact, the compression of nuclear matter at larger densities quite difficult. In ultra relativistic heavy-ion collision experiments the baryon densities can reach values of a few times $\rho_{0}$ at temperatures of $\sim 150-200 \mathrm{MeV}$. The only "natural laboratories" in which matter is compressed to densities up to ten times $\rho_{0}$ are compact stars. For these reasons, the study of these stellar objects can shed light on the equation of state of strongly interacting matter at extreme conditions. In particular, it has been extensively studied the possibility of formation, in the core of a compact star, of "exotic" particles as the hyperons or meson condensates of pions or kaons, or finally, it has been suggested that a phase transition from hadronic matter to quark matter, in which the quarks composing the baryons are deconfined, can occur. Concerning quark matter, in particular, recent studies on the QCD phase diagram at finite densities and temperatures have revealed the existence of a rich structure of the phase diagram with several possible phases in which a color superconducting state can be formed [3]. The fundamental phenomenological problem in the study of compact stars

\footnotetext{
a e-mail: pagliara@fe.infn.it
}

is to investigate how the measurable properties of these stellar objects as masses, radii, thermal evolution, periods of rotation etc., depend on the equation of state of matter. In principle, it is therefore possible to put constraints on the theory of the QCD phase diagram from astrophysical observations and measurements on compact stars. In this paper we will first analyze the equation of state of strongly interacting matter focusing in particular on the phase transition from hadronic matter to quark matter, eventually in its color superconducting phase and we will propose some signatures of the presence of quark matter in a compact star.

\section{Equation of state of compact stars matter}

\subsection{Hadronic matter}

Concerning hadronic matter, we use the relativistic fieldtheoretical approach to the nuclear equation of state [4]. In this theory the interactions between hadrons are described by the exchange of three mesons, the scalar field $\sigma$, the vector field $\omega$ and the isovector field $\rho$. The Lagrangian of the model has five free parameters which are fixed imposing that the model reproduces five measured quantities of the nuclear matter, i.e. the saturation density, the binding energy per nucleon, the incompressibility, the effective mass of nucleons and the symmetry energy. This model can easily incorporate all the particles of the baryon octet $[5,6]$ and in particular baryons containing 


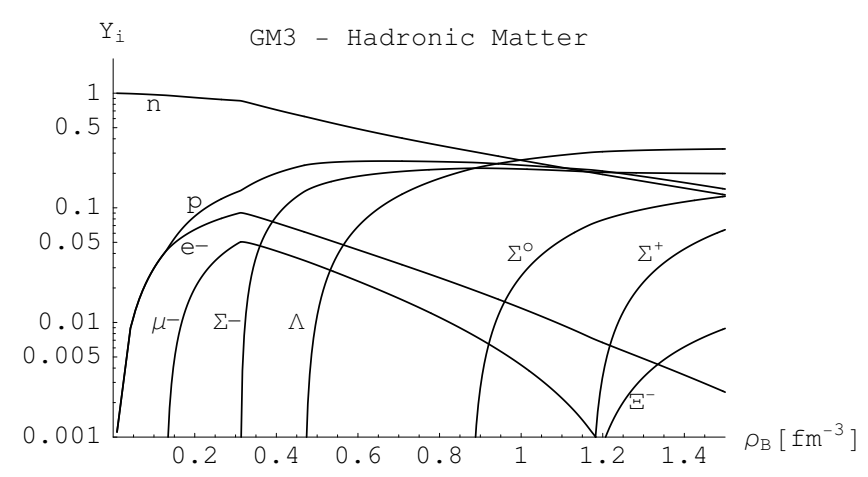

Fig. 1. Particle abundances as functions of the total baryon density.

strangeness, the hyperons. At $T=0$, in the mean-field approximation, the thermodynamic potential $\Omega$ per unit volume can be written as

$$
\begin{aligned}
\Omega= & -\frac{1}{3 \pi^{2}} \sum_{B} \int_{0}^{k_{F B}} \mathrm{~d} k \frac{k^{4}}{E_{B}^{\star}(k)}+\frac{1}{2} m_{\sigma}^{2} \sigma^{2} \\
& +\frac{1}{3} a \sigma^{3}+\frac{1}{4} b \sigma^{4}-\frac{1}{2} m_{\omega}^{2} \omega^{2}-\frac{1}{2} m_{\rho}^{2} \rho^{2},
\end{aligned}
$$

where the $\sum_{B}$ runs over the eight baryon species, $E_{B}^{\star}(k)=$ $\sqrt{k^{2}+M_{B}^{\star 2}}$ and the baryon effective masses are $M_{B}^{\star}=$ $M_{B}-g_{\sigma} \sigma$. The effective chemical potentials $\nu_{B}$ are given in terms of the thermodynamic chemical potentials $\mu_{B}$ and of the vector meson fields as follows:

$$
\nu_{B}=\mu_{B}-g_{\omega} \omega-t_{3 B} g_{\rho} \rho,
$$

where $t_{3 B}$ is the isospin 3 -component for baryon $B$ and the relation to the Fermi momentum $k_{F B}$ is provided by $\nu_{B}=\sqrt{k_{F B}^{2}+M_{B}^{\star 2}}$. The isoscalar and isovector meson fields $(\sigma, \omega$ and $\rho)$ are obtained as a solution of the field equations in the mean-field approximation [6].

The equation of state of compact star matter must satisfy the beta equilibrium and charge neutrality conditions. The former allows to express the chemical potentials of all the particles as linear combinations of the baryonic and electric charge chemical potential $\left(\mu_{B}\right.$ and $\left.\mu_{C}\right)$ :

$$
\mu_{i}=b_{i} \mu_{B}+c_{i} \mu_{C},
$$

where $b_{i}$ is the baryon number of the particle and $c_{i}$ is its electric charge in unit of the electron charge.

The condition of charge neutrality, taking into account also the densities of leptons, reads

$$
0=\rho_{p}+\rho_{\Sigma^{+}}-\rho_{\Sigma^{-}}-\rho_{\Xi^{-}}-\rho_{e}-\rho_{\mu},
$$

where $\rho_{i}$ indicate the number densities of the various particles. With all these conditions we can calculate the hadronic-matter equation of state as a function of only one chemical potential, the baryon chemical potential $\mu_{B}$.

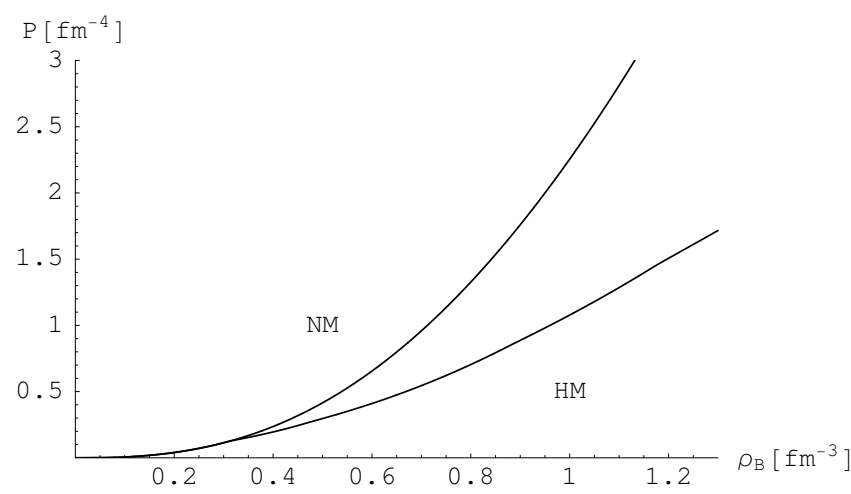

Fig. 2. Pressure as a function of baryon density for nucleonic matter (NM) and hadronic matter (HM).

In fig. 1 the particle abundances as functions of the total baryon density are displayed. Notice in particular how the density of neutrons start to decrease as neutral hyperons start to form. In fig. 2 the pressures as a function of baryon density for nuclear matter and hadronic matter are compared. As it can be seen the presence of hyperons makes the equation of state softer.

The model described so far will be used for densities of matter of the order of or larger than the saturation density $\rho_{0}$. For lower densities we will use the Negele-Vautherin and the Baym-Pethick-Sutherland equations of state $[1,2]$.

\subsection{Quark matter}

In high-density hadronic matter, baryons are forced to stay so close to one another that they would overlap, the constituent quarks are shared by neighboring baryons and should eventually become mobile over a distance larger than the typical size of one baryon. This means that quarks become deconfined and that at large densities they are the real degrees of freedom of strongly interacting matter instead of baryons. The process of deconfinement and the equation of state of quark matter can in principle be described by QCD. However in the energy scale involved in a compact star, QCD is non-perturbative and therefore simple phenomenological models are usually adopted to describe quark matter as the MIT bag model [7], the NJL model [8] or the Color Dielectric Model [9].

\subsubsection{Unpaired quark matter}

The simplest model to describe quark matter is the MIT bag model. In this model, quark matter is described as a gas of free quarks with massless up and down quarks and strange quarks having a "current mass" variable between $80-200 \mathrm{MeV}$. All the "non-perturbative physics of QCD" is simulated by the bag constant $B$ which represents the pressure of the vacuum. The thermodynamic 
potential reads

$$
\begin{aligned}
\Omega= & \frac{3}{\pi^{2}}\left(\int_{0}^{k_{F u}} \mathrm{~d} k k^{2}\left(k-\mu_{u}\right)+\int_{0}^{k_{F d}} \mathrm{~d} k k^{2}\left(k-\mu_{d}\right)\right. \\
& \left.+\int_{0}^{k_{F s}} \mathrm{~d} k k^{2}\left(\sqrt{k^{2}+m_{s}^{2}}-\mu_{s}\right)\right)+B
\end{aligned}
$$

where

$$
\begin{aligned}
k_{F u} & =\mu_{u}=\frac{1}{3} \mu_{B}+\frac{2}{3} \mu_{C}, \\
k_{F d} & =\mu_{d}=\frac{1}{3} \mu_{B}-\frac{1}{3} \mu_{C}, \\
k_{F s} & =\sqrt{\mu_{s}^{2}-m_{s}^{2}} \\
\mu_{s} & =\mu_{d},
\end{aligned}
$$

and $B$ is the bag constant. Beta stability is included in eqs. (6-9) and charge neutrality is imposed by the equation

$$
0=\frac{2}{3} \rho_{u}-\frac{1}{3} \rho_{d}-\frac{1}{3} \rho_{s}-\rho_{e}-\rho_{\mu} .
$$

As the hadronic equation of state, the unpaired quark matter equation of state has only one independent variable which can be chosen to be the baryon chemical potential $\mu_{B}$.

\subsubsection{Color superconducting quark matter}

Quark matter is actually a gas of interacting fermions, the interaction being mediated by the exchange of gluons as described by QCD. Considering just the one-gluon exchange potential, there is a channel of interaction between quarks which is attractive and which corresponds with the two incoming quarks to be in the $\overline{3}$ channel. From the results of the Bardeen-Cooper-Schrieffer theory of superconductivity, it is known that if in a Fermi gas there is an arbitrary weak attractive potential, the Fermi surface becomes unstable with respect to the formation of a condensate of Cooper pairs. Therefore, as it happens in metals at low temperature, also in quark matter an instability with respect to the formation of Cooper pairs between quarks does develop, originating the phenomenon of the so-called color superconductivity. The order parameter characterizing this phase is the value of the diquark condensate or, in other words, the color superconducting gap $\Delta$. The possibility of the existence of this phase of QCD was first shown at asymptotic densities, i.e. in a perturbative regime $[10,11]$. The same results have been extrapolated to lower densities using models of quark matter as for example the NJL model [12]. Both schemes leads to a value of $\Delta \sim 100 \mathrm{MeV}$. Such a high value indicates that, at variance with the superconductivity in metals, color superconductivity is very robust because in the only place of the universe in which it can appear, the core of compact stars, the temperature is very low (few keV). This observation has encouraged further studies in this direction, see ref. [3] for an exhaustive review.
It is not yet understood which type of color superconducting phase can appear in a compact star. It is widely accepted that the Color-Flavor Locking phase (CFL) is the real ground state of QCD at asymptotically large densities. In this phase, up, down, and strange quarks are present and all of them are involved in the formation of Cooper pairs. As a consequence, all the quarks have a common Fermi level and therefore charge neutrality and beta stability are automatically satisfied without the presence of leptons [13]. The CFL phase can form only if the mass of the strange quark $m_{s}$ is small with respect to the superconducting gap and the chemical potential. All these considerations are valid at high density. At lower densities, there is still uncertainty about the presence of CFL phase and in particular about the transition from the CFL phase to the hadronic phase. At intermediate values of $m_{s}$, it is in general difficult to involve strange quarks in BCS pairing due to their Fermi momentum, which is lower than that of up and down quarks. Recently, it has been shown that the CFL phase can form only if the ratio $m_{s}^{2} / \mu \lesssim 2 \Delta_{C F L}$ [14]. At larger values of $m_{s}^{2} / \mu$, but not too large values of $m_{s}$, the most energetically favored phase is the so-called gapless CFL (gCFL) phase instead of the $2 \mathrm{SC}$ phase or unpaired quark matter $[15,16,17]$. The gCFL phase has the same symmetries as the CFL phase but there are two gapless quark modes and a nonzero electron density and therefore it can have very different transport properties respect to the CFL phase.

To compute the equation of state of the (g)CFL phase we adopt the NJL-like formalism of refs. $[14,15,16]$ in which the thermodynamic potential per unit volume can be written as

$$
\begin{aligned}
\Omega= & -\frac{1}{4 \pi^{2}} \int \mathrm{d} p p^{2} \sum_{j}\left|\epsilon_{j}(p)\right| \rho_{j}(p) \\
& +\frac{1}{G}\left(\Delta_{1}^{2}+\Delta_{2}^{2}+\Delta_{3}^{2}\right)-\frac{\mu_{e}^{4}}{12 \pi^{2}},
\end{aligned}
$$

where $\Delta_{1}, \Delta_{2}, \Delta_{3}$ are the superconducting gaps characterizing the gCFL phase (which reduce to a single gap in the CFL phase), $G$ is the strength of the diquark coupling, $\epsilon_{j}(p)$ are the dispersion relations of quarks as in ref. [15] and $\mu_{e}$ is the electron chemical potential. Following the approximations used in refs. $[14,15]$, the effect of $m_{s}$ is introduced as a shift $-m_{s}^{2} / 2 \mu$ in the chemical potential for the strange quarks and the contributions of antiparticles is neglected. In eq. (11) we have introduced the quasiparticle probabilities

$$
\rho_{j}(p)=\frac{1}{2}\left(1-\frac{\tilde{\epsilon}_{j}(p)}{\epsilon_{j}(p)}\right),
$$

where $\tilde{\epsilon}_{j}(p)$ are the dispersion relations with vanishing gaps. To assure the convergence of the integral in eq. (11), a form factor $f=\left(\Lambda^{2} /\left(p^{2}+\Lambda^{2}\right)\right)^{2}$, which multiplies the gaps, is introduced in the dispersion relations $\epsilon_{j}(p)$. The form factor was fixed to mimic the effects of the asymptotic freedom of QCD [12] and the parameter $\Lambda$ was fixed at a value of $800 \mathrm{MeV}$. 


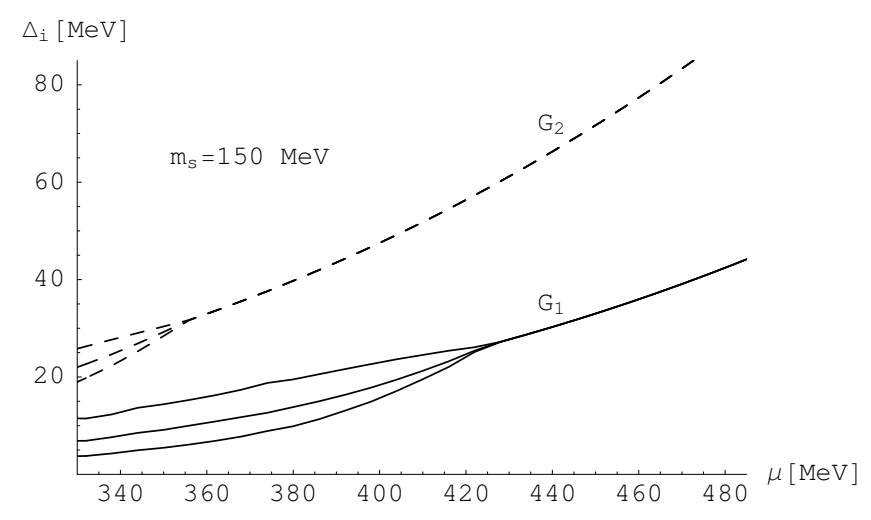

Fig. 3. Gap parameters as a function of the quark chemical potential for two different values of diquark coupling $G_{1}$ and $G_{2}$ and for a fixed value of the strange quark mass $m_{s}=150 \mathrm{MeV}$. The larger the value of $G$, the larger the window of the chemical potential in which the CFL phase occurs.

As in the other equations of state discussed so far we must impose the chemical equilibrium between the different quark species. The chemical equilibrium conditions (which also include $\beta$-stability) allow to express the chemical potential $\mu_{c f}$ ( $c$ and $f$ are the indexes of color and flavor, respectively) of each quark as a function of the quark (baryonic) chemical potential $\mu$, the electron chemical potential $\mu_{e}$ and the two chemical potentials, $\mu_{3}$ and $\mu_{8}$, associated to the $U(1) \times U(1)$ subgroup of the color gauge group (see ref. [15] for details). The color and electric charge neutrality are imposed by the following three equations:

$$
\frac{\partial \Omega}{\mu_{3}}=0, \quad \frac{\partial \Omega}{\mu_{8}}=0, \quad \frac{\partial \Omega}{\mu_{e}}=0 .
$$

Moreover, the thermodynamic potential must be minimized with respect to the gap parameters and therefore we have to impose the three additional conditions:

$$
\frac{\partial \Omega}{\Delta_{1}}=0, \quad \frac{\partial \Omega}{\Delta_{2}}=0, \quad \frac{\partial \Omega}{\Delta_{3}}=0
$$

The above equations allow us to compute the thermodynamic potential and all the thermodynamic variables as a function of the quark chemical potential only. In fig. 3 the gap parameters are displayed as functions of the quark chemical potential $\mu$ for two different values of the diquark coupling. $G_{1}$ and $G_{2}$ correspond, respectively, to values of $\Delta_{C F L} \sim 40$ and $\Delta_{C F L} \sim 100 \mathrm{MeV}$ at $\mu=500 \mathrm{MeV}$ and $m_{s}=150 \mathrm{MeV}$. It is interesting to observe that the window in which the gCFL phase appears depends noticeably on the value of the diquark coupling and, in particular, it decreases with $G$ (see fig. 3). This confirms the general argument for which the transition from gCFL to CFL occurs when $m_{s}^{2} / \mu \simeq 2 \Delta_{C F L}$.

\subsection{Phase transitions}

Although there is no theoretical evidence, it is believed that the hadronic matter-quark matter phase transition is
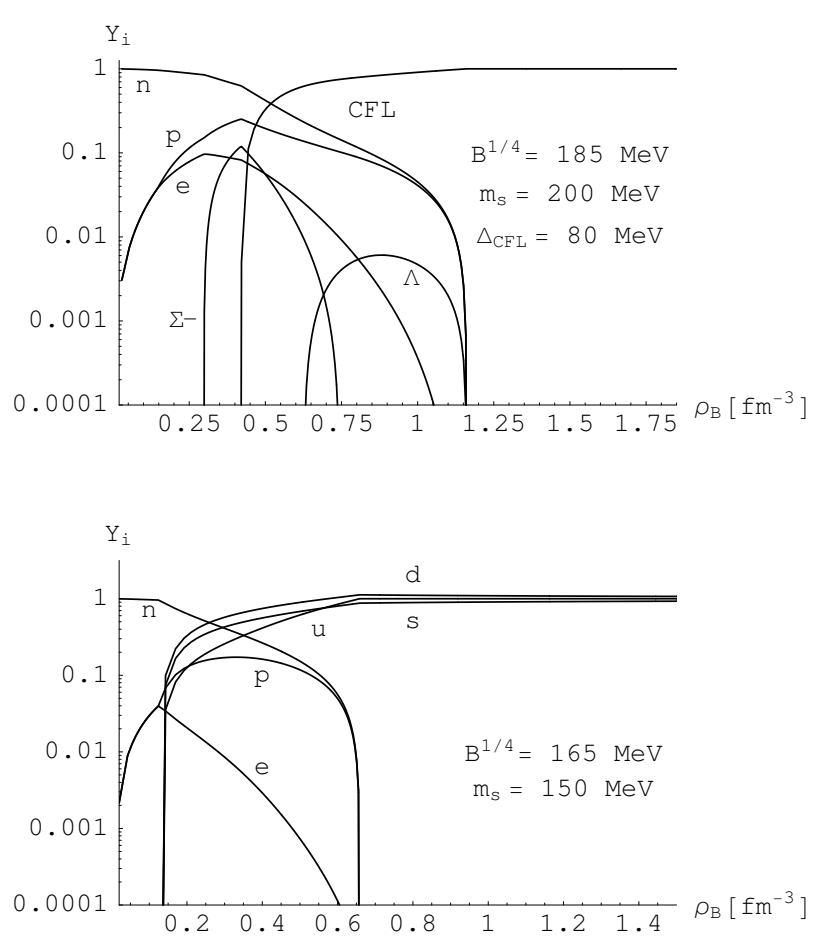

Fig. 4. Particle abundances as functions of the total baryon density. The upper panel corresponds to the case of the hyperon-quark mixed phase and the lower panel to the nucleonquark mixed phase.

a first order phase transition. Maxwell construction is the usual tool to connect two phases: the phase transition occurs at constant pressure with a discontinuity of the number density. In the context of compact stars, it has been shown that the two phases can be connected by an intermediate window of mixed phase [6]. In the calculation of the possible mixed phases, hadronic-unpaired quark phase or hadronic-CFL phase, we use the Gibbs construction in which the equations of mechanical, thermal and chemical equilibrium are simultaneously imposed. The conservation of the baryon number and the electrical neutrality are imposed as global conditions. Due to the existence of two conserved charges in the matter of a compact star the pressure need not to be constant in the mixed phase which is crucial for the stability of the star.

In fig. 4 the abundances of various particles as functions of the baryon density are shown for two different equations of state. In the upper panel, we show a mixed phase of hadronic matter and CFL quark matter where the superconducting gap is fixed at a value of $80 \mathrm{MeV}$. In the lower panel, we show a mixed phase of nuclear matter and unpaired quark matter.

The hypothesis of a direct transition from hadronic matter to the CFL phase is too simple at the light of recent results on the QCD phase diagram. Actually, a scenario in which an intermediate window of unpaired quark 


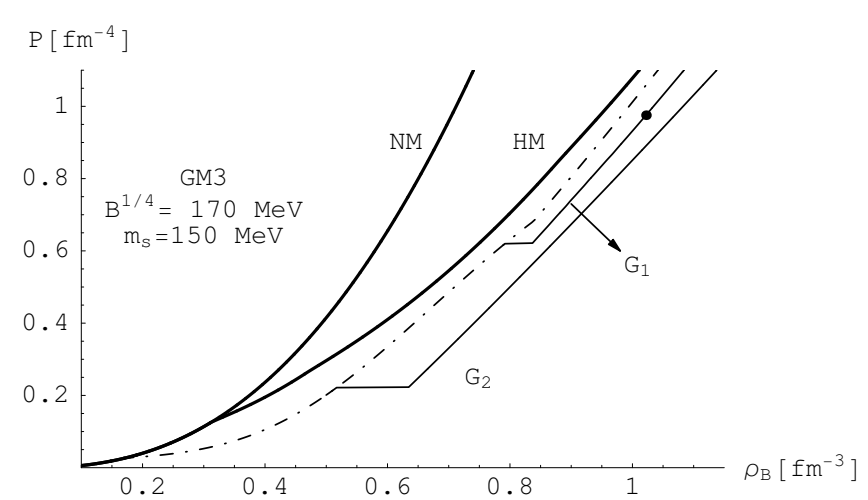

Fig. 5. Pressure as a function of baryon density for the scenario in which a first phase transition from hadronic matter to quark matter occurs via a mixed phase and then a second phase transition (here computed using the Maxwell construction) occurs from this mixed phase to the gCFL phase. The dot-dashed line is related to the hadron-unpaired quark mixed phase. The thin solid lines represent the phase transitions from the mixed phase to the gCFL phase for the two diquark couplings. Notice that in the case of $G_{1}$ a gCFL phase window is still present.

matter or 2SC quark matter is more plausible. In this case the equation of state of strongly interacting matter has two first-order phase transitions: a first one from hadronic matter to unpaired quark matter (or 2SC) and a second one from unpaired quark matter (or 2SC) to the (g)CFL phase (see refs. $[18,19,20])$. This scenario is shown in fig. 5 where the pressure as a function of the baryon density is shown for different equations of state. The first phase transition occurs between nuclear matter and unpaired quark matter (thick and dot-dashed lines) and the second phase transition (here computed using Maxwell construction for simplicity) occurs between the nuclear-quark mixed phase and the (g)CFL phase (dot-dashed and thin solid lines). The couplings $G_{1}$ and $G_{2}$ are the same of fig. 3 and the dot on the curve labeled with $G_{1}$ represents the onset of the CFL phase. Notice that for large diquark coupling $\left(G_{2}\right)$ the transition from the mixed phase to the (g)CFL phase occurs above the onset of the gCFL-CFL phase and for small diquark coupling $\left(G_{1}\right)$ a window of the gCFL phase is instead present. Concerning the hyperons, within this choice of parameters the transition from hadronic matter to gCFL quark matter occurs before reaching the threshold of the formation of hyperons. If we use larger values of the bag constant, the first critical density may be larger than the threshold of the formation of hyperons. In that case, however, the phase transition would involve the CFL phase directly.

\section{Signatures of quark matter in compact stars}

Several signatures of the presence of quark matter in compact stars have been proposed in the literature. The most extensively discussed is the mass-radius relation of compact stars which should allow the existence of very com-

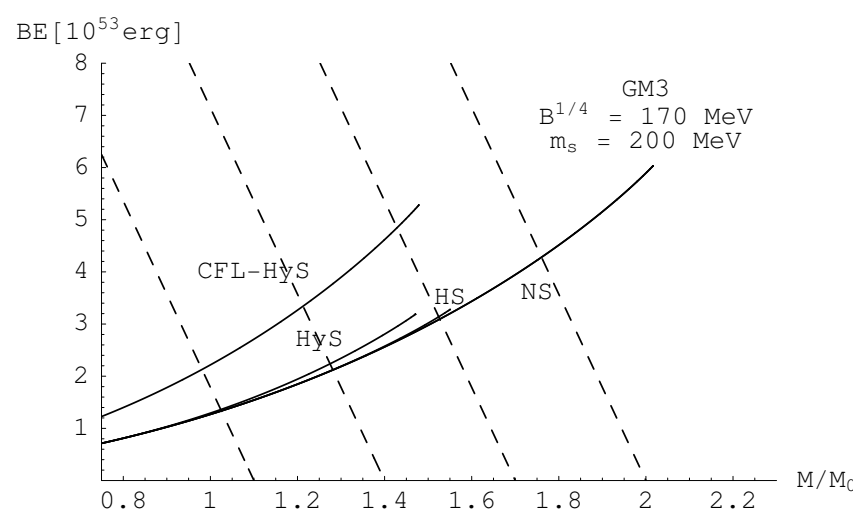

Fig. 6. The binding energies for neutron stars (NS), hadronic stars (HS), hybrid stars HyS (without CFL phase) and CFL hybrid stars (CFL-HyS) are shown as functions of the gravitational mass. The dashed line are the lines of constant baryonic mass.

pact stellar objects (radius less than $10 \mathrm{~km}$ ) if quark matter is present [21]. Other interesting ways to study the composition of a compact star come from the analysis of the cooling of the stars [22] or the stability with respect to the r-modes [23]. In both cases, the weak-decay channels involving the strange quark can strongly affect the transport properties of the matter of the compact star. Recently, it has been shown that also from gravitational wave signals from isolated compact stars, we can obtain important informations on the structure and composition of a star [24,25]. Finally, the formation of quark matter in a compact star can have a role also in the most violent explosive phenomena of the Universe, i.e. supernovae and gamma-ray-bursts [26]. Here, as an example, we will discuss only the effect of the formation of quark matter during a supernova explosion.

Let us first introduce the expression of the baryonic mass of a star:

$$
M_{B}=m_{n} \int_{0}^{R} \mathrm{~d} r \frac{4 \pi r^{2}}{(1-2 m(r) / r)^{1 / 2}} \rho_{B}(r),
$$

where $m_{n}$ is the neutron mass, $m(r)$ is the mass in a sphere of radius $r, \rho_{B}$ is the total baryon density. The energy released in a supernova explosion is the difference between the gravitational mass of the core of the progenitor star $M_{c}$ and the gravitational mass of the final compact star. The energy released corresponds also to the binding energy of the star. For practical purposes we can assume that $M_{c} \sim$ $M_{B}$. Therefore, the energy released can be approximated as the difference $M_{B}-M$. Almost all of this energy is released in neutrinos. In fig. 6 , we display the binding energy for neutron stars, hadronic stars and hybrid stars (without CFL) and CFL-hybrid stars, as functions of the gravitational mass. For a fixed value of the baryonic mass, which as already stated roughly corresponds to the mass of the collapsing core, we can calculate the energy released in the collapse. Typical values of this energy are of the order of $10^{53}$ ergs. It is evident from fig. 6 that, for a fixed baryonic mass (dashed lines), the binding energy of a CFL-hybrid 
star can be a few times that of a neutron star and therefore the corresponding energy released is larger. Figure 6, suggests, moreover, that, if in the future new supernova events will be detected, it will be possible from the signal of the emitted neutrinos to obtain information on the equation of state of the matter in compact stars. For instance, if the energy released in neutrinos is of the order of or larger than $\sim 4 \times 10^{53} \mathrm{erg}$, we can exclude hybrid or hadronic stars. A similar energy may be released in the formation of a neutron star but this would require a baryon mass for the core $\sim 2 M_{\odot}$ what seems difficult in the light of recent supernovae simulations [27]. In this case only a CFL (CFLhybrid) star can be a plausible candidate. This conclusion agrees with the well-known results that the stars containing quark matter are more bounded that hadronic stars.

\section{Conclusions}

In this paper, we studied the equation of state of strongly interacting matter at large densities and vanishing temperature. We analyzed in particular the possibility of a phase transition from hadronic matter and quark matter. We included in our calculation the recent results on the QCD phase diagram indicating two first-order phase transitions: a first one from hadronic matter to unpaired quark matter and a second one from unpaired quark matter to the color flavor locked phase. These theoretical results can be tested studying compact stars which are the only places in the universe in which the density of matter can reach values up to ten times the nuclear matter saturation density. We listed the different signatures of the presence of quark matter in a compact star and we discussed, in particular, a scenario in which during a supernova explosion deconfinement of quarks is realized in the newly born compact star.

\section{References}

1. G. Baym, C. Pethick, P. Sutherland, Astrophys. J. 170, 299 (1971).

2. J.W. Negele, D. Vautherin, Nucl. Phys. A 207, 298 (1973).
3. K. Rajagopal, F. Wilczek, hep-ph/0011333 (2000).

4. B.D. Serot, J.D. Walecka, Phys. Lett. B 87, 172 (1979).

5. N.K. Glendenning, S.A. Moszkowski, Phys. Rev. Lett. 67, 2414 (1991).

6. N. Glendenning, Compact Stars (Springer-Verlag, 1997).

7. G. Baym, S.A. Chin, Phys. Lett. B 62, 241 (1976).

8. K. Schertler, S. Leupold, J. Schaffner-Bielich, Phys. Rev. C 60, 025801 (1999).

9. A. Drago, U. Tambini, M. Hjorth-Jensen, Phys. Lett. B 380, 13 (1996).

10. B.C. Barrois, Nucl. Phys. B 129, 390 (1977).

11. D. Bailin, A. Love, Phys. Rep. 107, 325 (1984).

12. M.G. Alford, K. Rajagopal, F. Wilczek, Nucl. Phys. B 537, 443 (1999).

13. K. Rajagopal, F. Wilczek, Phys. Rev. Lett. 86, 3492 (2001).

14. M. Alford, C. Kouvaris, K. Rajagopal, Phys. Rev. Lett. 92, 222001 (2004).

15. M. Alford, C. Kouvaris, K. Rajagopal, Phys. Rev. D 71, 054009 (2005).

16. K. Fukushima, C. Kouvaris, K. Rajagopal, Phys. Rev. D 71, 034002 (2005).

17. S.B. Ruster, I.A. Shovkovy, D.H. Rischke, Nucl. Phys. A 743, 127 (2004).

18. S.B. Ruster, V. Werth, M. Buballa, I.A. Shovkovy, D.H. Rischke, hep-ph/0503184 (2005).

19. D. Blaschke, S. Fredriksson, H. Grigorian, A.M. Oztas, F. Sandin, hep-ph/0503194 (2005).

20. A. Lavagno, G. Pagliara, nucl-th/0504066 (2005).

21. A. Drago, A. Lavagno, G. Pagliara, Phys. Rev. D 69, 057505 (2004).

22. H. Grigorian, D. Blaschke, D. Voskresensky, Phys. Rev. C 71, 045801 (2005).

23. A. Drago, A. Lavagno, G. Pagliara, Phys. Rev. D 71, 103004 (2005).

24. N. Andersson, D.I. Jones, K.D. Kokkotas, Mon. Not. R. Astron. Soc. 337, 1224 (2002).

25. A. Drago, G. Pagliara, Z. Berezhiani, gr-qc/0405145 (2004).

26. Z. Berezhiani et al., Astrophys. J. 586, 1250 (2003).

27. S.E. Woosley, A. Heger, T.A. Weaver, Rev. Mod. Phys. 74, 1015 (2002). 